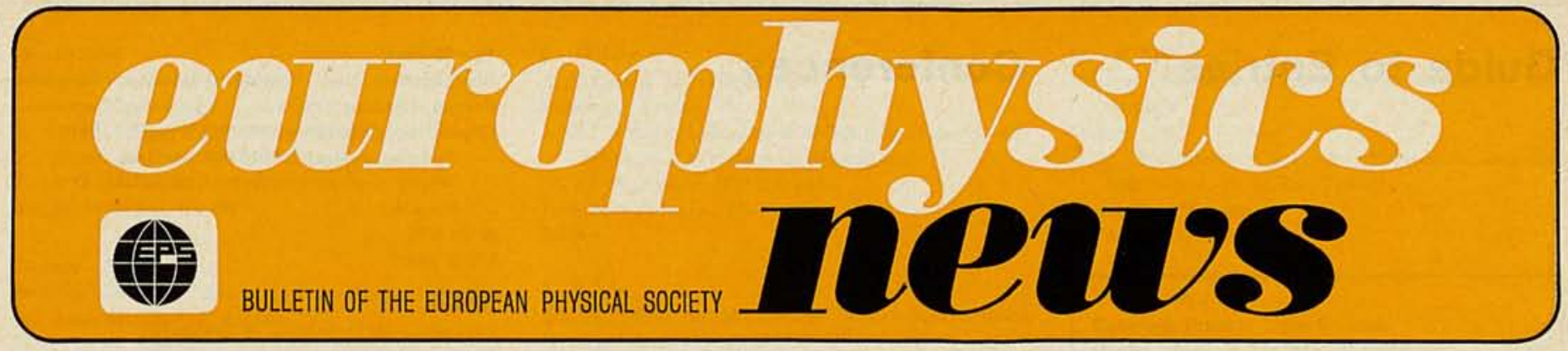

\title{
EPS Conference Committee
}

The Conference Committee, set up to advise the Executive Committee of the European Physical Society on all matters regarding scientific meetings, has met three times since its formation early in 1969. Basic guidelines have been formulated concerning the various categories of conference and the criteria for sponsorship by the EPS. The EPS Divisions act in an advisory capacity to the Conference Committee for specific fields of physics.

A different task of the Conference Committee, which has been entrusted to a special study group, is to define the framework of the next general conference similar to the Inaugural Conference held in April 1969 in Florence. Another study group is investigating the organization of conferences restricted to a few participants and devoted to a well-defined specific topic. Such conferences, on the lines of the famouis Gordon Conferences held in the USA, are given the title of Europhysics Conferences and were described in Europhysics News No. 7, January 1970.

Medium-size topical conferences are organized by the EPS Divisions. The European character of these conferences is guaranteed by the criteria established by the Conference Committee. Interference with the international conferences which are sponsored by IUPAP is avoided.

Any conference held in Europe which satisfies the following conditions is eligible for sponsorship by the EPS:

\section{Scientific Value}

a) There should be a clearly demonstrated need for the proposed conference, i.e. new and important advances since the last conference of a similar type took place. b) The invited speakers and the papers accepted for discussion should be of high calibre.

c) Acceptance of communications should be based on some sort of refereeing system which ensures a level comparable with that of papers in the regular journals.

2. International Character

a) There should be an international committee to advise on the scientific programme and on the names of the lecturers. With the agreement of the International Advisory Committee the detailed organization and the refereeing of communications may be in the hands of a local organizing committee. The refereeing of communications and the editing of the published proceedings should, however, have an international element.

b) Participation should be genuinely international, both as regards participants and invited speakers. The aim should be that at least one half of the invited speakers, rapporteurs and discussion leaders should come from outside the host country.

c) Attendance must be independent of nationality or citizenship. The International Advisory Committee should in addition ensure an appropriate geographical distribution of participants.

d) The EPS will only sponsor conferences on the understanding that for the venue chosen there will be no restriction on the attendance of members from anywhere (By-laws, Rule 32). e) EPS sponsorship relates to meetings with a predominant European character.

\section{Organization}

Sponsorship is granted by the Executive Committee of the European Physical Society on the recommendation of the Conference Committee.

All requests for sponsorship should be addressed to the EPS Secretariat in Geneva, twelve months prior to the date of the conference concerned, as follows:

\section{Conference Committee, European Physical Society, PO Box, CH-1227 Carouge, Geneva, Switzerland.}

The EPS endeavours to collect information about physics conferences and summerschools as far in advance as possible in order to bring it to the notice of physicists in Europe. This is done by publication of meetings lists in the normal issues of Europhysics News. It has been decided, in addition, to publish twice a year a "Meetings Issue" of Europhysics News listing all forthcoming conferences known to the EPS including those previously announced. The Main Secretariat wishes to thank all those who have provided information to help compile this first Meetings Issue. 


\section{Guide to Entries}

In this first Meetings Issue of Europhysics News, details are given of all future meetings about which notification has been received up to the end of May 1970. The events are arranged in date order, with summerschools being listed separately. The meetings organized by Divisions of the EPS are printed in bold type and boxed; those sponsored by the EPS are printed in bold type. Europhysics Conferences are indicated by a star following the date. In general, meetings are open to any applicants: where attendance is known to be restricted to invited applicants this is indicated accordingly.

The information is given in the following order:

Date

Title

Venue

Contact for information

(A) Application deadline / (P) Deadline for papers / Number of participants / By invitation only (inv.)

Language, if English is not used / Sponsorship / Conference fee

\section{Conferences}

\section{0}

$10-12$ June

Bubble-Chamber Technology

Argonne, Illinois, USA

M. Derrick, High Energy Physics Division, Argonne National Laboratory, Argonne, III. 60439

13 - 14 June

The Role of Scientific Research in the Contemporary Society

Florence, Italy

Italian Physical Society,

Via L. degli Andalò 2, I-40124 Bologna Italian / free

\section{4 - 18 June}

Nuclear Science and Engineering

Los Angeles, California, USA

D.G. Pettingill, American Nuclear Society, 244 East Ogden Ave., Hinsdale; III. 60521

14- 19 June

18th Annual Conference on Mass Spectrometry and Allied Topics

San Francisco, California, USA

R.E. Honig, RCA Laboratories, Princeton N.J. 08540

\section{5 - 17 June}

Applied Superconductivity

Boulder, Colorado, USA

S. Autler, NASA Electronics Research Center, Code RRA, Cambridge, Mass. 02139

P: 1.3 .70

\section{5 - 17 June}

Electron Beam Welding and Smelting

Paris, France

M. Rapin, C.E.A., Service MA.

Boite postale No 61, F-92 Montrouge

15 - 19 June *

Atomic Transport in Solids and Liquids

Marstrand, Sweden

A. Lodding, Arkitektvägen 6B, S-43080 Hovas

A: $15.2 .70 /$ P: 15.4 .70

$100 /$ S.Kr. 360

\section{5 - 19 June}

International Symposium on Information Theory Noordwijk, The Netherlands

Meetings Information, IEEE, 345 E 47th St., New York, N.Y. 10017

\section{5 - 19 June}

Nuclear Data for Reactors

Helsinki, Finland

C. Lathwell, Ministry of Technology,

Millbank Tower, London SW 1

A: $15.6 .70 /$ inv.

\section{5 - 19 June}

Vacuum Metallurgy

Anaheim, California, USA

L.M. Bianchi, TRW Inc., 23555 Euclid Ave., Cleveland, Ohio 44117
15 - 20 June

Identification and Process Parameter Estimation Prague, Czechoslovakia

Organizing Committee of 2nd Prague IFAC Symposium, Institute of Information

Theory and Automation, Vyšehradská 49,

Prague 2

A: 31.5 .70

US $\$ 40-45$

16 June

Crystallographic Studies at Cryogenic Temperatures

London, UK

The Meetings Officer, IPPS, 47 Belgrave Square, London SW 1

17 June

Electron and lon Scattering at a Surface

Abingdon, UK

The Meetings Officer, IPPS, 47 Belgrave Square, London SW 1

17 - 19 June

Cryogenic Engineering

Boulder, Colorado, USA

R.A. Cliffe, National Academy of Sciences, 2101 Constitution Ave. N.W., Washington, D.C. 20418

\section{8 - 19 June}

Symposium on Feedback and Dynamic Control of Plasmas

Princeton, N.J., USA

T.K. Chu, Secretary, Plasma Physics Laboratory, Princeton University, РОВ 451, Princeton, N.J. 08540

P: 15.5 .70

\section{2 - 24 June}

American Physical Society, Canadian Association of Physicists, Sociedad Mexicana de Fisica, Joint Meeting

Winnipeg, Canad

W.W. Havens jr., American Physical Society 335 E. 45th St., New York, N.Y. 10017

22 - 24 June

Future of Diagnostic Radiology

London, UK

R.E. Steiner c/o CIBA Foundation, 41 Portland Place, London WIN 4 BN

22 - 25 June

Symposium on the Effects of Radiation on Structural Materials

Toronto, Canada

A.L. Bement, Battelle Memorial Institute, Pacific Northwest Lab., Box 999 Richland, Washington, 99352

22 - 27 June

Workshop on Communication Theory and Some Applications

Sorrento, Italy

E. Corti, Istituto Elettrotecnico, Via Claudio 21, 1-80125 Napoli

A: 30.4 .70

22 - 27 June

7 th International Symposium on the Chemistry of Natural Products

Riga, USSR

S.N. Ananchenko, Inst. for Chemistry of Natural Products, Academy of Sciences.

ul. Vavilova 32, Moscow

IUPAC

\section{June}

Theory of Magnetism in Metals

Oxford, UK

The Meetings Officer, IPPS, 47 Belgrave Square, London SW 1

23 June - 4 July

Interaction of Energetic Charged Particles with Solids

Ankara, Turkey

National Accelerator Laboratory, POB 500, Batavia, III, 60510, USA 


\section{4 - 26 June}

Anelastic Properties of Solids

Lausanne, Switzerland

B. Vittoz, Ecole Polytechnique Fédérale,

33, av. de Cour, $\mathrm{CH}-1007$ Lausanne

A: 1.5 .70 / P. $1.4 .70 / 100$

Sw. frs 50 to $S w$. frs 100

\section{June - 2 July}

American Nuclear Society, 16th Annual Meeting Los Angeles, California, USA

R.R. Quinn, Meetings Manager, American Nuclear Society, 244 East Ogden Ave., Hinsdale,

III. 60521

\section{June -4 July}

Radiation Research

Evian, France

Secrétariat du IVe Congrès international de Radiobiologie, c/o Wagons-Lits Cook, 16 , rue du Mont-Blanc, CP 230,

IUPAP $\mathrm{CH}-1211$ Genève

\section{9 - 30 June}

Optimization Techniques in Circuit and Control Applications

London, UK

The Conference Dept., IEE, Savoy Place, London WC 2

\section{June - 1 July}

\section{Microscopy}

Chicago, Illinois, USA

Inter Micro-70, McCrone Research Institute, 451 East 31 St., Chicago, III. 60616

29 June - 1 July

Symposium of Engineering Applications of Lasers

Tel Aviv, Israe

U. Shimony, Dept. of Electrical Engineering, Technion, Haifa.

\section{June - 3 July}

Symposium on the De Sitter and Conformal Groups

Boulder, Colorado, USA

A.O. Barut, Department of Physics, University of Colorado, Boulder, Colorado 80302

\section{July}

Electrical Measurement of the Chemica Properties of Sea Water

Washington, D.C., USA

A.E. Wheeler, North American Rockwell Corp. Space Div., 12214 Lakewood Blvd.

Downey, Calif. 90241

\section{5- 6 July}

Annual Meeting of the Israel Physical Society

Tel Aviv, Israel

A.J. Greenfield, Secretary, Israel - Physical Society, Bar - Ilan University, Ramat-Gan

\section{5 - 9 July}

Advanced Measurement and Contro

Cardiff, UK

O.G. Pamely-Evans, University of Wales, Dept. of Applied Physics, Institute of Science and Technology, Cathays Park, Cardiff

\section{6 - 9 July}

International Conference on Ferrites

Kyoto, Japan

Mitsui Sugimoto, Institute of Physical and

Chemical Research, Yamato-Machi, Saimata Prefecture

\section{6 - 10 July}

Microwave Spectroscopy

Bangor, UK

The Meetings Officer, IPPS, 47 Belgrave Square, London SW 1

A: $1.5 .70 /$ P: 16.1 .70

\section{6 - 11 July}

Applications of Holography

Besançon, France

J. Ch. Viénot, Laboratoire d'Optique, Faculté des Sciences, Université de Besançon

La Bouloie, F-25 Besançon

$P$ : Before January / 500

IUPAP

\section{7 - 9 July}

Scanning Electron Microscopy in Materials Science Newcastle-upon-Tyne, UK

The Meetings Officer, IPPS, 47 Belgrave Square, London SW 1

P: 1.3 .70

7 - 10 July

Chemistry and Physics of Organic Scintillation and Liquid Scintillation Counting

San Francisco, California, USA

D.L. Horrocks, Chemistry Div., Argonne National Laboratory, 9700 South Cass Ave. Argonne, III. 60439

7 - 11 July

7th International Colloquium of Corpuscular Photography and Visual Solid Detectors Barcelona, Spain

Secrétariat du Laboratoire de Physique corpusculaire, Centre de Recherches nucléaires, rue du Loess, F-67 Strasbourg-Cronenbourg

\section{8 - 11 July}

Atomic Structure Theory

Paris and Orsay, France

CNRS, 15, quai Anatole-France, F-75 Paris 7e

\section{3 - 15 July}

Annual Meeting, American Association of Physicists in Medicine

Washington, D.C., USA

J.A. Hancock jr., Box 2546 Malcolm Grow USAF Med. Ctr., Andrews AFB, Washington

\& 15 D.C. 20331

\section{3 - 17 July}

Working Seminar on the Role of History of Physics in Physics Education

Cambridge, Massachusetts, USA

A.L. King, Dartmouth College, Hanover, New UPAP

\section{4 - 17 July \\ 2nd Conference on Atomic Spectroscopy Hannover, Federal Republic of Germany \\ A. Steudel, Institut für Experimentalphysik A, Technische Universität Hannover, Welfen- garten 1, D-3 Hannover \\ P: $20.5 .70 / 250$ \\ DM 20.-}

15 - 17 July

Testing Fibrous Composites for Mechanical Properties

Teddington, UK

The Meetings Officer, IPPS, 47 Belgrave Square, London SW 1

P: 27.2 .70

20- 24 July

Dielectric Materials, Measurements and Applications

Lancaster, UK

The Meetings Officer, IPPS, 47 Belgrave Square, London SW 1

\section{1 - 24 July}

Nuclear and Space Radiation

San Diego, California, USA

R.A. Poll, Systems, Science and Software, POB 1620, La Jolla, Calif, 92037

\section{1 - 24 July}

Second International Conference on Atomic Physics

Oxford, UK

G.K. Woodgate, Dept. of Physics, Clarendon Laboratory, Parks Rd., Oxford

$P: 1.4 .70 / 200 /$ inv.

IUPAP / \& 19.10 .0

\section{2 - 24 July *}

\section{Coherent Tunable Light Sources}

Munich, Federal Republic of Germany

J. Schubert, Siemens AG., Research Laboratories 43 , Hofmannstr. 51, D-8000 München 25

27 - 31 July

29th Research Conference on Instrumentation Science

Geneva, New York, USA

N.E. Huston, University of Wisconsin, 1815 University Ave., Madison, Wisc. 53706

\section{8 - 30 July}

Nonaqueous Solvents

Manchester, UK

J.F. Gibson, The Chemical Society,

Burlington House, London WIV OBN

\section{2- 7 August}

High Speed Photography

Denver, Colorado, USA

Society of Motion Picture and Television

Engineers, 9 East 41 Street,

New York, N.Y. 10017

\section{3 - 7 August}

Precision Measurements and Fundamental Constants

Gaithersburg, Maryland, USA

L.M. Branscomb, A 363 Physics Building, National Bureau of Standards, Washington, D.C. 20234

IUPAP, IUPAC

\section{4- 11 August}

The Motion, Evolution of Orbits, and Origin of Comets

Leningrad, USSR

G.A. Chebotarev, Institute for Theoretical Astronomy, Mendelejevkaja Linia 1 , Leningrad B-164

\section{6 - 7 August}

The Crab Nebula

Jodrell Bank, UK

F. Graham Smith, Nuffield Radio Astronomy Lab., Jodrell Bank Macclesfield, Cheshire IAU

\section{0 - 14 August}

External Galaxies and Quasi Stellar Objects Uppsala, Sweden

M. Schmidt, Mount Wilson and Palomar Observatories, 1201 East California Boulevard, Pasadena, Calif. 91106 inv.

IAU

\section{0 - 14 August}

4th Nordic Solid State Conference

Turku, Finland

V. Hovi, Wihuri Physical Laboratory,

University of Turku, Turku 2

A: $1.6 .70 /$ P: $1.5 .70 / 400$

\section{0 - 15 August}

New Techniques in Space Astronomy Munich, Federal Republic of Germany Max-Planck-Institut für Physik und Astrophysik, Institut für Extraterrestrische Physik, D-8046 Garching/München

A: $15.3 .70 / P: 1.6 .70 / 150 /$ inv.

$\mathrm{IAU} / \& 5$ 
10 - 15 August

White Dwarfs

St. Andrews, UK

W.J. Luyten, 221 Space Science Building, University of Minnesota, Minneapolis, IAU Minn. 55455

\section{1 - 14 August}

International Federation of Automatic Control, Symposium

Kyoto, Japan

Dept. of Electronics, Faculty of Engineering, University of Kyoto, Yoshida-Honmachi, Sakyo-Ku, Kyoto

\section{$12-14$ August}

Automation in Optical Astrophysics

Edinburgh, UK

Astronomer Royal for Scotland, Department of Astronomy, Royal Observatory, Edinburgh 9 A: $30.4 .70 / \mathrm{P}: 12.8 .70 / 50 /$ inv. IAU

\section{2 - 14 Augus}

Gravitational N-Body Problem

Cambridge, UK

G. Contopoulos, University of Thessaloniki, Thessaloniki, Greece

P: $31.5 .70 / 50 /$ inv.

IAU

\section{2 - 15 August}

The IAU-System of Astronomical Constants Heidelberg, Federal Republic of Germany G.A. Wilkins, H.M. Nautical Almanac Office,

Royal Greenwich Observatory, Herstmonceux

Castle, Hailsham, Sussex, UK

$30 /$ inv

IAU

\section{7 - 21 August}

Angular Correlations in Nuclear Disintegration Delft, The Netherlands

B. van Nooijen, Technische Hogeschool, Dept. of Experimental and Nuclear Physics,

Berlageweg 15, Delft

$170 /$ inv.

IUPAP / approx. $\$ 20$

\section{7 - 21 August}

Semi-Conductors

Boston, Massachusetts, USA

E.W.J. Mitchell, Secretary, Semi-Conductors

Commission, J.J. Thomson Physical Laboratory, IUPAP

Whiteknights Park, Reading, Berkshire, UK

\section{$18-21$ August}

Microelectronics Circuits and Systems Theory Sydney, Australia

K.G. Knight, Secretariat for International Conference on Microelectronics, Institution of Radio and Electronics Engineers (Austr.),

Science House, 157 Gloucester Street, Sydney, NSW, 2000

\section{4 - 26 Augus}

Radiation Damage

Albany, New York, USA

E.W.J. Mitchell, Secretary, Semi-Conductors Commission, J.J. Thomson Physical Laboratory, IUPAP

Whiteknights Park, Reading, Berkshire, UK

\section{4 - 28 August}

3rd International Liquid Crystal Conference

Berlin, Federal Republic of Germany

R. Hosemann, Fritz-Haber Institut der Max-Planck-

Gesellschaft, Faradayweg 4-6,

$$
\text { D-1 Berlin } 33
$$

P: 1.5 .70

DM 70.-

\section{6 - 29 Augus}

Small Angle X-Ray Scattering

Graz, Austria

O. Kratky, Institute for Physical Chemistry University of Graz, A-8010 Graz
26 August - 4 September

15th Int. Conference on High Energy Physics Kiev, USSR

N.N. Bogolubov, Joint Institute for Nuclear Research, Head Post Office, IPAP Box 79 , Moscow

\section{7 - 29 August}

Transport Properties of Solids

Sydney, Australia

G.K. White, CSIRO, Division of Physics, Sydney University Grounds, Chippendale, NSW 2008, IUPAP

28 August - 2 September

7th International Mineralogical Association Congress

Tokyo and Kyoto, Japan

Agency of Industrial Science and Technology, Geological Survey of Japan, 8 Kawada-cho, Ichigaya, Shinjuku-ku, Tokyo

30 August - 5 September

4th International Congress of Electron Microscopy

Grenoble, France

Société Française de Physique, 33, rue Croulebarbe, F-75 Paris 13 e

\section{August - 4 September}

Heat Transfer

Paris/Nersailles, France

Société Française des Thermiciens

28, rue de la Source, F-75 Paris $16 e$ 1000

\& 80 until 1 June $1970 / \& 100$ after 1 June 1970

\section{August - 4 September}

Hybrid Computation

Munich, Federal Republic of Germany

J. Heinhold, Kongressbüro AICA - IFIP 1970,

Institut für Angewandte Mathematik der Technischen Hochschule München, Arcisstr. 21 D-8 München 2

A. $15.4 .70 / P$. $15.4 .70 /$ inv.

US $\$ 55$

\section{August - 4 September}

Polarisation Phenomena in Nuclear Reactions Madison, Wisconsin, USA

H.H. Barschall, University of Wisconsin,

Department of Physics, Madison, Wisc. 53706

A: $1.8 .70 / P: 1.7 .70 / 250 /$ inv.

IUPAP / \& 20

\section{August - 4 September}

Solar Magnetic Fields

Paris, France

R. Howard, Hale Observatories, 813 Santa Barbara Street, Pasadena, Calif. 91106 P: $15.4 .70 / 125 /$ inv.

IAU

\section{August - 4 September}

Triennial International Conference on Mass Spectroscopy

Brussels, Belgium

J. Drowart, Conference Vice-Chairman, Faculty of Sciences, University of Brussels,

Avenue F.-D. Roosevelt, B-1050 Brussels 5 A: $31.7 .70 / P: 5.1 .70 / 800$

$\$ 25$

31 August - 4 September

2nd European Symposium on Cosmic Rays: Modulation Effects

Amsterdam, The Netherlands

M. Arens, Natuurkundig Laboratorium, Universiteit van Amsterdam, Valckenierstraat 65 ,

Amsterdam-C

P: $15.5 .70 / 70$

FI. 35.-
31 August - 4 September *

4th European Conference on Controlled

Fusion and Plasma Physics

Rome, Italy

B. Brunelli, Laboratori Gas-Ionizzati,

C.P. 65. 1-00044 Frascati

A: $1.6 .70 /$ P: $1.6 .70 / 400$

Lire 13000

\section{1 - 3 September}

Nuclear Power in 1970

Williamsburg, USA

W. Thomas, Virginia Electronics and Power,

Richmond, Va, 23219

P: $15.4 .70 / 400$

\section{1- 3 September}

Techniques for the Measurement of Fundamental Spectroscopic Data

London, UK

W.R.S. Garton, Department of Physics, Imperial College, Prince Consort Road, London SW 7 P: $1.5 .70 / 100 /$ inv.

$\mid A U / £ 5$

\section{1- 4 September}

Discharges and Electrical Insulation in Vacuum Waterloo, Ontario, Canada

K.D. Srivastava, Dept. of Electrical Engineering, University of Waterloo, Waterloo IUPAP

\section{4 September}

1st International Conference on the Hydraulic Transport of Solids in Pipes

Coventry, UK

Conference Secretary, BHRA, Cranfield, Bedford A: $20.8 .70 / 250 /$ inv.

\section{1- 5 September}

Magnetic Resonances and Related Phenomena

Bucharest, Rumania

V. Lupei, Institute for Atomic Physics, РОВ 35 , Bucharest

A: $10.5 .70 /$ P: $15.5 .70 / 1000$

US $\$ 30$

\section{2- 4 September}

Man-Computer Interaction

Teddington, UK

The Conference Dept., IEE, Savoy Place, London WC 2

\section{4- 10 September}

12th International Conference on Low Temperature Physics, LT12

Kyoto, Japan

Organizing Committee for LT 12, Science Council of Japan, Ueno Park, Tokyo 110

A: $1.5 .70 / P: 1.4 .70 / 800 /$ inv.

IUPAP / 5000 yen (US \& 13.75)

\section{6 - 11 September}

Hyperfine Interactions Detected by Nuclear Radiation

Rehovoth, Israel

G. Goldring, Nuclear Physics Department Weizmann Institute of Science, Rehovoth A: $1.4 .70 / P: 15.7 .70 / 140$

IUPAP / \$ 25

\section{6 - 11 September}

Symposium on Hadron Spectroscopy

Balatonfüred, Hungary

G. Marx, Institute for Theoretical Physics, Eötvös Lorand University, Puskin Utca 5 , Budapest VIII

A: $15.6 .70 /$ P: $15.6 .70 / 100$

$\$ 55$

6 - 12 September

Electron Microscopy

Manchester, UK

The Meetings Officer, IPPS, 47 Belgrave Square, London SW 1 
6 - 15 September

4th International Conference on the Peaceful Uses of Atomic Energy

Geneva, Switzerland

United Nations, Palais des Nations,

$\mathrm{CH}-1200$ Geneva

\section{7 - 9 September}

EUCHEM Conference on Electron Spectroscopy

Uppsala, Sweden

O. Nilsson, Secretary, Institute of Physics Box 530, S-751 21 Uppsala

\section{7 - 9 September}

Ion Implantation

Reading, UK

The Meetings Officer, IPPS, 47 Belgrave Square, London SW

A: June 1970

\section{7 - 9 September}

2nd Annual Conference on Computational Physics London, UK

The Meetings Officer, IPPS, 47 Belgrave Square, London SW 1

P: 7.9 .70

\section{7 - 10 September}

6th Annual International Quantum Electronics Conference

Kyoto, Japan

Institute of Electronics and Communication Engineers of Japan, Kikai Shinko Kaikan Shiba Park, Minato-ku, Tokyo

\section{7 - 11 September}

Microwave and Optical Generation and Amplification

Amsterdam, The Netherlands

Conf. Orgn. MOGA 70, Postbus 341, Eindhoven

\section{7 - 11 Septembe}

2nd International Codata Conference

St. Andrews, UK

N.H. Robinson, British National Committee on Data for Science and Technology, The Roya Society, 6 Carlton House Terrace, London SW 1

\& 30 and \& 17.10. for meals and accommodation

\section{7 - 11 September}

6th International Congress on Cybernetics

Namur, Belgium

Secretariat of the Intern. Assoc. for Cybernetics, Palais des Expositions,

Place André-Rijckmans, Namur

\section{8 - 10 September}

Optical Techniques for Investigations in the Upper Atmosphere

Exeter, UK

The Meetings Officer, IPPS, 47 Belgrave Square London SW 1

\section{8 - 12 September}

Molecular Structure and Spectroscopy

Columbus, Ohio, USA

K.N. Rao, Department of Physics, Ohio State University, 174 West 18th Ave., Columbus, Ohio 43210

\section{9 - 12 September}

Sagamore Conference III on Charge, Spin and Momentum Density

Aussois, France

E.F. Bertaut, Rayons-X, Laboratoire d'Electrostatique et de Physique du Métal, rue des Martyrs, Cedex No. 166, F-38 Grenoble-Gare

$80 /$ inv.

IUPAP/IUCr / \$ 50

\section{0 - 11 September}

International NEVAC Symposium on Surface

Physics and Ultra-High Vacuum Science and Technology

Groningen, The Netherlands

S.H.A. Begemann, Laboratorium voor Technische Natuurkunde, Rijksuniversiteit Paddepoelcomplex, Groningen

A: $1.7 .70 / P: 1.4 .70 / 200$

FI. 75.-

\section{0 - 11 September}

Magnetic Resonance in Non-Conductors

Nottingham, UK

S. Clough, Physics Department, University of Nottingham, Nottingham, NG7 2 RD

A: $30.6 .70 / P: 30.6 .70 / 140$

\section{1 - 17 September}

International Congress on the Education of

Teachers of Physics in Secondary Schools

Eger, Hungary

D. Sette, Università di Roma, Facoltà di Ingegneria, Istituto di Fisica, I-Roma

A: $15.5 .70 / P: 15.5 .70 / 150 /$ inv.

IUPAP $/ \$ 20$

\section{September}

Chemical Physics on Surfaces

Chicago, Illinois, USA

G.A. Somorjei, University of California

Department of Chemistry, Berkeley, Calif. 94720

\section{4 - 15 September}

H.S. Harned Memorial Symposium Electrolyte Solutions

Chicago, Illinois, USA

M.H. Lietzke, Oak Ridge National Laboratory, Oak Ridge, Tenn. 37830

US $\$ 25$

14- 16 September

Photoionization Phenomena and Photoelectron Spectroscopy

Oxford, UK

The Meetings Officer, IPPS, 47 Belgrave Square, London SW 1

14 - 17 September

Corrosion Inhibitors

Ferrara, Italy

V. Carrasiti, Centro di Studi sulla Corrosione, Aldo Deceo, Via Scanidiana, Ferrara

\section{4 - 18 September}

The Physics of Non-Crystalline Solids III

Sheffield, UK

B. Ellis, Department of Glass Technology, University of Sheffield, Northumberland Road, Sheffield S10 2TZ

A: $31.7 .70 / 200$

\& 35

\section{4 - 18 September}

2nd International Symposium on Organic Solid. State Chemistry

Rehovoth, Israel

M.D. Cohen, The Weizmann Institute of Science, Rehovoth

P: $31.7 .70 / 60.80 /$ inv

IUCr, IUPAC / \$ 25

\section{4 - 19 September}

International Conference on Magnetism

Grenoble, France

R. Pauthenet, Laboratoire d'Electrostatique et de Physique du Métal, Cedex No 166, F-38 Grenoble-Gare

P: $1.4 .70 / 1000 /$ inv.

IUPAP / FF 150.-, with Proceedings FF 250.-

\section{4 - 22 September}

XIIIth International Conference on Coordination Chemistry

Cracow-Zakopane, Poland

B. Jezowska-Trzebiatowska, Institute of Chemistry, University of Wroclaw, PI. Uniwersytecki 1, Wroclaw

A: $30.9 .69 / \mathrm{P}: 30.3 .70 / 1000 /$ inv

IUPAC / US \& 25

\section{5 - 18 Septembe}

Electric Discharges in Gases

London, UK

Conference Department, IEE, Savoy Place, London WC 2

inv.

\section{5 - 18 September}

4th Annual Solid State Devices Conference

Exeter, UK

The Meetings Officer, IPPS, 47 Belgrave Square, London SW

P: 19.6 .70

\section{8 - 19 September}

Solid State Physic

Bucharest, Rumania

Institutul de Fizica Atomica, Magurele, Bucharest

\section{8 - 20 September}

Effects of Nuffield A-level on Higher Education Science Courses

Birmingham UK

The Meetings Officer, IPPS, 47, Belgrave Square, London SW 1

\section{1 - 23 September}

Magnetic Thin Films

Prague, Czechoslovakia

L. Valenta, Department of Theoretical Physics, Charles University, $\mathrm{Ke}$ Karlovu 3, Prague 2 A: $15.4 .70 / P: 1.9 .70 / 200 /$ inv.

IUPAP / US \& 25

\section{1 - 23 September}

Temperature Measurement

Warwick, UK

The Meetings Officer, IPPS, 47, Belgrave Square, London SW 1

\section{1 - 24 September}

Engineering in the Ocean Environment

Panama City, Florida, USA

C.B. Koesy, IEEE Oceanography Co-ordination Committee, IEEE, Panama City, Fla.

A: March

\section{1 - 25 September}

Autumn Meeting of the Austrian Physical Society Vienna, Austria

K. Lintner, Strudlhofgasse 4, A-1090 Vienna

A: $21.9 .70 / P: 30.6 .70 / 250$

German / OS 50.-

\section{1 - 26 September}

Processus électroniques simples et multiples du domaine $X$ et $X$-UV

Paris, France

M.F. Wuilleumier, Lab de Chimie Physique,

1, rue Pierre-et-Marie-Curie, F-75 Paris $5 e$

P: $1.5 .70 / 50 /$ inv

IUPAP

\section{2 - 24 September}

Vitreous State

Bristol, UK

The Faraday Society, 6 Gray's Inn Square London WC 1

A: $15.9 .70 / 150-200 /$ inv.

\& 5

\section{3 - 25 September}

Gaussian Wave Functions

Nottingham, UK

R.L. Hudson, Department of Mathematics,

University of Nottingham, University Park

Nottingham NG7 2RD

A: $7.7 .70 / P: 16.8 .70 / 90 /$ inv.

\& 9

\section{3 - 25 September}

High Energy Physics

Durham, UK

The Meetings Officer, IPPS, 47, Belgrave Square, London SW

A: July $70 /$ P: 1.8 .70

\section{3 - 25 September}

Stress Analysis Today

Guildford, UK

The Meetings Officer, IPPS, 47, Belgrave Square, London SW 1

P: 20.4 .70 


\section{3 - 26 September}

Crystal Growth and Epitaxy from the Vapour Phase Zurich, Switzerland

E. Kaldis, Laboratorium für Festkörperphysik, ETH, Gloriastr. 35, $\mathrm{CH}-8006$ Zurich

A: $15.6 .70 / P: 6.4 .70 / 150$

Sw. frs 100.-

28 - 30 September

Int. Conference on Metastable Alloys

Brela, Yugoslavia

Conference on Metastable Alloys, Institute of Physics of the University, РОВ 02-816,

Zagreb, Yugoslavia

A: February $1970 /$ P: June $1970 / 80$

IUPAP / \& 10

28 - 30 September

Thin Liquid Films and Boundary Layers

Cambridge, UK

The Faraday Society, c/o B.A. Pethica, Unilever Research Laboratory, Port Sunlight, Wirral, Cheshire

A: $21.9 .70 / 200-250 /$ inv.

\section{September - 3 October}

35. Physikertagung

Hannover, Federal Republic of Germany

K.H. Riewe, Deutsche Physikalische

Gesellschaft $\Theta . V$.

Heraeusstr. 9, D-645 Hanau

2000-2500

DM 30.- member, DM 75.- non-members

\section{September - 2 October}

Conférence sur la physique des hautes énergies

Aix-en-Provence, France

MMe M.S. Detoeuf, Institut du Radium,

11, rue Pierre-et-Marie-Curie, F-75 Paris 5e

P: $1.7 .70 / 400 /$ inv.

French / FF 20.- (for non-members of French Physical Society)

\section{September}

Erosion of Surfaces and Sputtering

Crawley, UK

The Meetings Officer, IPPS, 47, Belgrave Square, London SW 1

\section{3 October}

5th Symposium on Thermophysical Properties

Newton, Massachusetts, USA

C.F. Bonilla, Engineering School, Columbia $\$ 40$

University, New York, N.Y. 10017

\section{4- 9 October}

17th International Union of Biological Sciences, General Assembly

Washington, D.C., USA

F.A. Stafleu, Botanical Museum, Lange

Nieuwstr. 106, Utrecht, The Netherlands

\section{5- 7 October}

Gallium Arsenide and Related Compounds

Aachen, Federal Republic of Germany

The Meetings Officer, IPPS, 47, Belgrave Square, London SW 1

\section{5- 10 October}

International Congress on Thin Films

Cannes, France

Mme J. Fauvet-Mainier, Société Française des Ingénieurs et Techniciens du Vide,

600 19, rue du Renard, F-75 Paris 4 e

FF 200.- member SFITV, FF 250.- non-member SFITV

\section{6 - 9 October}

4th International Conference on Plutonium and Other Actinides

Santa $\mathrm{Fe}$, New Mexico, USA

F.W. Schonfeld, Los Alamos Scientific Laboratory, Los Alamos, New Mexico 87544
7 - 9 October

Microwave Power Symposium

Scheveningen, The Netherlands

H.A. Püschner, $\mathrm{CH}-5314$ Kleindöttingen

A: $31.5 .70 /$ P: $31.5 .70 / 200-300$

\$ 25

\section{1 - 17 October}

The Physics and Chemistry of Semiconductors, Heterojunctions and Layer Structures

Budapest, Hungary

G. Gergely, Vice President of the Organizing Committee, РОВ 76, Budapest IV, Ujpest 1

P: 1.7 .70

IUPAP / \$ 45

\section{6 - 18 October}

Autumn Meeting of Swiss Physical Society

Basel, Switzerland

F. Kneubühl, Physik-Institut, ETH,

Gloriastr. $35, \mathrm{CH}-8006$ Zürich

\section{9 - 24 October}

Material Testing

Budapest, Hungary

Scientific Society of Mechanical Engineers, Szabadsàg tér 17, Budapest V

\section{$19-24$ October}

Statistical Mechanics

Mexico

E. Braun \& A. Morales, Comision Nacional de Energia Nuclear, Av. Insurgentes Sur 1079, Apartado Postal No 27-190, Mexico 18, D.F. IUPAP

\section{6 - 28 October}

17th Spectroscopy Symposium and Exhibition of Instrumentation

Ottawa, Canada

R. Ironside, Applied Chemistry Division, National Research Council, Ottowa 7

P: $24.5 .70 / 200$

Canadian Dollars 15

\section{8 - 29 October}

Radiation and Isotope Techniques in the Building Industry

Brussels, Belgium

Eurisotop Office, Commission of the European Communities, Berlaymont Building, 200, rue de la Loi, B-1040 Brussels

P: 15.3 .70

\section{8 - 30 October}

International Electron Devices Meeting

Washington, D.C., USA

Meetings Information, IEEE, $345 \mathrm{E}$. 47th St., New York, N.Y. 10017

\section{October - 3 November}

National Meeting of the Italian Physical Society

Venice, Italy

Italian Physical Society, Via L. degl Andald 2, 1-40124 Bologna

A: $11.9 .70 /$ P: $11.9 .70 / 600-800 /$ inv.

Italian / Lire 1000, Lire 3000

\section{6 November}

Nuclear Science Symposium

New York, N.Y., USA

Meetings Information, IEEE, $345 \mathrm{E}$. 47th St., New York, N.Y. 10017

\section{5 - 19 Novembe}

American Nuclear Society and Atomic Industrial Forum, Joint Meeting and Atomic Fair

Washington, D.C., USA

R.R. Quinn, Meetings Manager, American Nuclear Society, 244 East Ogden Ave., Hinsdale, III. 60521

\section{5 - 19 November}

Magnetism and Magnetic Materials Los Angeles, Calif., USA

Meetings Information, IEEE, $345 \mathrm{E}$. 47th St., New York, N.Y. 10017
17 - 19 November

Fall Joint Computer Conference

Houston, Texas, USA

AFIPS HDaS, 210 Summit Ave.,

Montvale, N.J. 07645

17 - 21 November

Laser Plasma

Moscow, USSR

O.N. Krokhin, P.N. Lebedev Physics Institute, Academy of Sciences of the USSR,

Leninskiy Prospekt 53, Moscow B-312

P: June / $150 /$ inv.

\section{3 - 27 November}

Symposium on New Developments in Physical and Biological Radiation Detectors

Vienna, Austria

H.H. Storhaug, Division of Scientific and Technical Information, IAEA, Kärntnerring 11, POB 590, A-1011 Vienna

P: $10.7 .70 /$ inv.

14 - 15 December

Optical Studies of Adsorbed Layers

London, UK

The Faraday Society, 6 Gray's Inn Square, London WC 1

100-150 / inv.

L 3

14 - 18 December

Relativistic Astrophysics

Austin, Texas, USA

L.C. Shepley, Physics Department, University of Texas, Austin, Texas 78712

\section{1}

5 - 7 January

8th Annual Solid State Physics Conference Manchester, UK

The Meetings Officer, IPPS, 47 Belgrave Square London SW 1

\section{6 March}

Nuclear and High-Energy Physics

Würzburg, Federal Republic of Germany

U. Schmidt-Rohr, POB 1248, D-69 Heidelberg

P: $10.12 .70 / 600-700$

members approx. DM 20.-

\section{8 - 13 March}

High Polymer Physics, Molecule Physics

Berlin, Federal Republic of Germany

Professor Dottahn, Abbestr, 2-12, D-1 Berlin 10

P: $10.12 .70 / 200-250$

members approx. DM 20.-

\section{5 - 20 March}

Extraterrestrial Physics, Mass Spectroscopy, High Frequency Physics

Bad Nauheim, Federal Republic of Germany

D. Kossel c/o Ernst Leitz GmbH., D-633 Wetzlar

P: $15.12 .70 / 300$

members approx. DM 20.-

\section{2 - 27 March}

Semi-Conductor Physics, Metal Physics, Low

Temperatures, Thermodynamics

Münster, Federal Republic of Germany

D. Kamke, University, D-463 Bochum

P: $15.12 .70 / 500-700$

members approx. DM 20.-

\section{3 - 25 March}

Electro-Optics International

Brighton, UK

G. Johns, Press Officer, Trident Publicity,

14 Queen Anne Terrace, North Hill,

Plymouth PL4 8EG

\section{P: 15.8 .70}

\section{3 - 26 March}

Negative lons

Liverpool, UK

The Meetings Officer, IPPS, 47 Belgrave Square, London SW 1

P: 2.1 .71 


\section{3 - 26 March}

5th International Conference on Fluid Sealing Reading, UK

Conference Secretary, BHRA, Cranfield, Bedford A: February 1971 / P: $30.9 .70 / 250 /$ inv.

\& 30

\section{9 - 31 March}

Magnetism

Salzburg, Austria

K.H. Riewe, Deutsche Physikalische

Gesellschaft e.V.,

Heraeusstr. 9, D-645 Hanau

P: $15.12 .70 / 200-250$

members approx. DM 20.-

\section{March - 3 April}

Acoustics, High Speed Physics

Ulm, Federal Republic of Germany

F. Fränz c/o AEG-Telefunken, D-79 Ulm

$P: 20.12 .70 / 200$

members approx. DM 20:-

5 - 8 April

3rd National Atomic and Molecular Physics Conference

York, UK

The Meetings Officer, IPPS, 47 Belgrave Square, London SW 1

19 - 23 April

5th Conference on Magnetohydrodynamic Electrical Power Generation

Munich, Federal Republic of Germany

J. Faure (Mme), ENEA, 38, boulevard Suchet,

F-75 Paris $16 e$

A: $1.1 .71 / \mathrm{P}: 1.10 .70 / 400$

May

5th International Symposium "Photon Detectors"

Varna, Bulgaria

E. Djakov, Bulgarian Academy of Sciences, ul. "7 Noemvri' 1, Sofia

$60 /$ inv

US $\$ 10$

\section{5 - 29 May}

Journées de Physique de 1971

Evian, France

Société Française de Physique

33, rue Croulebarbe, F-75 Paris $13 e$ 100

French / FF 20

\section{5 - 7 July}

Meeting on Rare Earths and Actinides

Durham, UK

Solid State Physics Sub-Committee, IPPS,

47, Belgrave Square, London SW 1

\section{5 - 8 July}

8th International Shock Tube Symposium

London, UK

J.L. Stollery, Symposium Secretary, Department of Aeronautics, Imperial College,

Prince Consort Road, London SW 1

P: title 1.9.70, manuscript: 1.1 .71

\section{5 - 9 July}

3rd International Conference on Crystal Growth Marseille, France

B. Mutaftschiev, Université d'Aix-Marseille - CNRS, Saint-Jérôme 13, F-13 Marseille 13

IUCr, IUPAP, IUPAC

\section{8 - 11 July}

Nuclear Three Body Problem

Budapest, Hungary

Eötvös Lorand Physical Society,

Szabadsàg-tér 17, Budapest $\mathrm{V}$

A: $1.5 .71 / P: 1.6 .71 / 150 /$ inv.

$\$ 10$

\section{$19-23$ July}

Light Scattering in Solids

Paris, France

M. Balkanski, Laboratoire de Physique des Solides,

Tour 13, 2e étage, Faculté des Sciences,

9, quai Saint-Bernard, F-75 Paris $5 e$

A: $15.1 .71 / P: 15.2 .71 / 200$

IUPAP / \& 50
25 - 31 July

23rd International Congress of Pure and Applied Chemistry

Boston, Massachusetts, USA

XXIII Congress of Pure and Applied Chemistry, A.T. Winstead, Director c/o American

Chemical Society, 1155 Sixteenth Street N.W. Washington, D.C. 20036

IUPAC

23 - 27 August

Statistical Properties of Complex Nuclear Systems

New York, N.Y., USA

J.B. Garg, SUNY, Albany, N.Y. 12203

\section{1 - 3 September}

Pattern Recognition

Brighton, UK

The Meetings Officer, IPPS, 47 Belgrave Square, London SW 1

12 - 18 September

EPS Solid State Physics Conference

Florence, Italy

A.B. Lidiard, AERE Building 89, Harwell Didcot, Berkshire, UK .

13 - 18 September

Symposium on Dynamics of lonized Gases Tokyo, Japan

Professor Sato, Institute of Space and Aeronautical Sciences, University of Tokyo, Komaba, Meguro-ku

$100 /$ inv.

IUTAM

13 - 18 September

10th International Conference on Phenomena in onized Gase Oxford, UK

The Meetings Officer, IPPS, 47 Belgrave Square, London SW 1

20 - 23 September

2nd European Meeting on Ferroelectricity

Dijon, France

L. Godefroy, Faculté des Sciences de Dijon, Boulevard Gabriel 6, F-21 Dijon

A: $14.7 .71 / P: 1.6 .71 / 200$

$\mathrm{IUCr} / \mathrm{FF} 80$

\section{1 - 23 September}

Modern Developments in Flow Measuremen Harwell, UK

F.K. Pyne, AERE, Harwell, Didcot, Berkshire

A: 2.7.71/P: $1.3 .71 / 150 /$ inv

$£ 15-£ 20$

\section{2 - 24 September}

Nuclear Physics Sub-Committee Meeting

Oxford, UK

L.L. Green, Oliver Lodge Laboratory, University of Liverpool, POB 147, Liverpool L69 3BX

\section{2}

August - September

9th General Assembly and Congress of IUC

Kyoto or Tokyo, Japan

IUCr

September

Molecular Spectroscopy

Wroclaw, Poland

Institute of Low Temperatures and Structural Researches, Polish Academy of Sciences,

Wroclaw, Katedralny 1 , Wroclaw

P. $30.6 .70 / 150 /$ inv

US $\$ 15$

\section{5 - 12 September}

Nuclear Structure Study with Neutrons

Balatonfüred, Hungary

D. Kiss, Executive Secretary of the Organizing Committee, Central Research Institute of 300 Physics, POB 49, Budapest 114

UPAP
6- 12 September

European Congress on Electron Microscopy (EMCON 72)

Manchester, UK

The Meetings Officer, IPPS, 47 Belgrave Square, London SW 1

\section{Summerschools}

\section{0}

\section{2 - 25 June}

High Energy Scattering of Composite Objects

Zakopane, Poland

E. Obryk, Institute of Nuclear Physics Cracow 23

A: 30.4 .70

15 - 29 June

International School of Atmospheric Physics Trapani, Italy

M.T. Tibaldi (Miss), Istituto di Fisica,

Via Irnerio 46, 1-40126 Bologna

A: $31.3 .70 /$ inv.

\section{6 - 26 June}

Summerschool on Lasers and Laser Applications Haifa, Israe

S. Kimel, Dept, of Chemistry,

Technion, Israel Institute of Technology, Haifa

21 June - 5 July

1970 CERN School of Physics

Loma-Koli, Finland

W.O. Lock, Personnel Division, CERN $\mathrm{CH}-1211$ Geneva 23

A: $15,3.70$

22 June - 4 July

Summerschool on Low Temperature Physics

Twente, The Netherlands

W.J. Huiskamp, Kamerlingh Onnes Laboratorium, Nieuwsteeg 18, Leiden

29 June -4 July

Summerschool on the Electrical Properties of Non-Crystalline Material Cambridge, UK

R.P. Ferrier, Cavendish Laboratory, Free School Lane, Cambridge CB2 3 RQ

A: 13.4 .70

29 June - 10 July

Health Physics Summerschool 1970

London, UK

H.D. Evans, Dept. of Chemical Engineering and Chemical Technology, Imperial College of Science and Technology, London SW 7

29 June - 11 July

Foundations of Quantum Mechanics

Varenna, Italy

F. Selleri, Istituto di Fisica, Via Amendola 173, 1-70126, Bar A: $30.4 .70 / 40$

\section{June - 25 July}

Quantum Field Theory: Renormalization and Non-Perturbative Approximations Cargese, Corsica, France

Laboratoire de Physique Théorique, Faculté des Sciences de Paris Tour 22, 9, quai Saint-Bernard, F-75 Paris $5 e$

A: 15.3 .70

1- 15 July

Summerschool on Crystal Optics of Absorbing Materials

Orléans, France

M.M. Lévy, Lab. de Crystallographie et Minéralogie, Faculté des Sciences, Tours 16-26, 9, quai Saint-Bernard F-75 Paris $5 e$ 
1- 19 July

Elementary Processes at High Energy

Trapani, Italy

G. Preparata, The Rockefeller University New York, N.Y. 10021 USA

A: $1.5 .70 /$ inv.

\section{July - 29 August}

Mathematical Physics, Quantum Field

Theory and Statistical Mechanics

Les Houches, France

Ecole d'été de Physique théorique, F-74 Les Houches

$$
\text { A: } 15.3 .70
$$

FF 1200

\section{6 - 17 July}

Plasma Physics Summerschool

Abingdon, UK

Summerschool Office, Room 140, Building ES,

Culham Laboratory, Abingdon, Berkshire

\section{A: $18.5 .70 /$ inv.}

\&2

\section{9 - 23 July}

Nuclear Reactions at Low Energies

Predeal, Rumania

A. Corciovei, Institute for Atomic Physics, POB 35 Bucharest

A: 15.5 .70

\section{3 - 25 July}

Mantle and Core in Planetary Physics

Varenna, Italy

Società Italiana di Fisica, Via L. degli Andalò 2, I-40124 Bologna

A: 30.4 .70

L 80000

20 - 31 July

Heidelberg-Karlsruhe International Summer

Institute in Theoretical Physics -

Strong Interaction Physics

Heidelberg, Federal Republic of Germany

H.D. Dahmen, Institut für Theoretische Physik,

Philosophenweg 16, D.69 Heidelberg

\section{1 - 31 July}

Hadron Interactions

Bagur, Spain

H.H. Ostermann (Miss), Secretary,

Instituto de Fisica Teorica,

Avda. José Antonio, 585 Barcelona 7

A: 1.5 .70

\section{July - 15 August}

Scottish Universities Summerschool on Hadronic Interactions of Electrons and Photons

Edinburgh, UK

I.M. Barbour, Department of Natural Philosophy, The University, Glasgow W 2

26 July - 29 August

Quantum Chemistry, Solid State Physics and Quantum Biology

Uppsala, Sweden and

Beitostolen, Norway

Quantum Chemistry Group, Uppsala University, POB 518, S-751 20 Uppsala

A: $1.5 .70 / 130 /$ inv.

$\$ 510$

27 July - 8 August

Critical Phenomena

Varenna, Italy

Società Italiana di Fisica, Via L. degli Andalò 2 1-40124 Bologna

A: $30.4 .70 /$ inv.

L 80000

\section{$10-21$ August}

Mathematical Physics

Istanbul, Turkey

A.O. Barut, Department of Physics,

University of Colorado, Boulder

Colorado 80302 , USA

$$
\text { A: } 1.6 .70
$$

\section{0 - 21 August}

Theory of Strong Interaction and its Implication to Weak and Electromagnetic Processes

Copenhagen, Denmark

N. Brene, Niels Bohr Institute, Copenhagen

\section{7 - 28 August}

Summerschool in Medium Energy Nuclear Physics

Banff, Canada

G.C. Neilson, Nuclear Research Centre, University of Alberta, Edmonton, Canada

\section{7 - 29 August}

XV International Summer Meeting of Nuclear Physicists on Nuclear Structure

Herceg-Novi, Yugoslavia

A.H. Kukoc, Institute "Boris Kidrich", POB 522, Belgrade

A: $15.5 .70 / 100 /$ inv.

US $\$ 100$

26 August - 2 September

Applications of Magnetic Resonance in Biology Oxford, UK

R.E. Richards, Physical Chemistry Laboratory, South Parks Road, Oxford OXI 3 QZ

30 August - 12 September

1970 CERN Computing and Data Processing School

Varenna, Italy

Scientific Conference Secretariat, CERN, CH-1211 Geneva 23

A: 1.3 .70

Sw. frs 700.-

\section{August - 9 September}

Magnetism

La Colle sur Loup, Nice, France

S. Foner, Francis Bitter National Magnet Laboratory, Massachusetts Institute of Technology, 170 Albany Street, Cambridge, Mass. 02139

\section{August - 12 September}

Theoretical Principles of Heterogenous Catalysis on Semi-Conductors

Constance, Federal Republic of Germany

Institut für Physikalische Chemie,

Robert-Mayer-Str. 11, D-6 Frankfurt/Main A: 31.5 .70

\section{August - 26 September}

Revision of Physics for Teachers in Secondary Modern Schools

Erice, Italy

V. Adragna, Biblioteca Comunale, 1-91016 Erice (Trapani) inv.

\section{7 - 12 September}

Activation Analysis in Geochemistry and Cosmochemistry

Kjeller, Norway

E. Andersen, Reactor School, Institutt for Atomenergi, POB 40, $\mathrm{N}-2007$ Kjeller

A: 1.5 .70
7 - 19 September

Direct and Patterson Methods of Solving

Crystal Structures

Parma, Italy

M.M. Woolfson, Department of Physics, University of York, Heslington, York, UK A: $1.4 .70 / 80 /$ inv.

$\$ 25$

\section{September - 3 October}

Autumn School for High Energy Physics in Germany 1970

Maria Laach, Federal Republic of Germany

I. Dammann, Deutsches Elektronen-Synchrotron, DESY, Notkestieg 1, D-2000 Hamburg 52

A: 29.5 .70 (qualification required: 50

$\mathrm{PhD}$ in Experimental High Energy Physics)

German

12 - 16 Octobe

Superconductivity

Pegnitz, Federal Republic of Germany

R. Gremmelmaier, Siemens AG., Forschungs laboratorien, Günther-Scharowsky-Str. 2, D.852 Erlangen

A: $29.5 .70 / 60 /$ inv.

German

\section{3- 27 October}

Plasma Physics

Telavi, USSR

H.L. Tsintsadze, Institute of Physics of the Georgian Academy of the Sciences of the USSR, Tbilisi

\section{1}

July

Interaction of Energetic Charged Particles with Solids

Ankara, Turkey

E. Inonü, Middle East Technical University, Ankara

\section{2}

July

Plasma Physics Summerschool

Les Houches, France

J.L. Delcroix, Laboratoire de Physique des Plasmas, F-91 Orsay
Published by the European Physical Society

Correspondence to: EUROPHYSICS NEWS European Physical Society P.O. BOX $\mathrm{CH}-1227$ Carouge Genève Switzerland

Editorial Advisory Panel: G.-J. Béné, L. Jansen, E.N. Shaw, L. EtienneAmberg

Editor: W.S. Newman

Printed by: Ed. Cherix \& Filanosa S.A. $\mathrm{CH}-1260$ Nyon, Switzerland 